\title{
MESH ADAPTATION USING ADJOINT METHODS AND REDUCED-ORDER MODELS FOR LARGE EDDY SIMULATION
}

\author{
Xiaodong $\mathrm{Li}^{1}$, Steven Hulshoff ${ }^{2}$ and Stefan Hickel ${ }^{3}$ \\ Delft University of Technology, Kluyverweg 2, 2629 HS Delft, Netherlands \\ ${ }^{1}$ X.Li-12@tudelft.nl, ${ }^{2}$ SJHulshoff@tudelft.nl, ${ }^{3}$ S.Hickel@tudelft.nl
}

Key words: Computational Fluid Dynamics, Large Eddy Simulation, Adaptive Mesh Refinement, Adjoint Method, Posterior Error Estimation, Reduced-Order Model

\begin{abstract}
Adaptive mesh refinement (AMR) is potentially an effective way to automatically generate computational meshes for high-fidelity simulations such as Large Eddy Simulation (LES). Adjoint methods, which are able to localize error contributions, can be used to optimize the mesh for computing a physical quantity of interest (e.g. lift, drag) during AMR. When adjoint-based AMR techniques are applied to LES, primal flow solutions are needed to solve the adjoint problem backward in time due to the nonlinearity of Navier-Stokes equations. However, the resources required to store primal flow solutions can be huge, even prohibitive, in practical problems because of the long averaging time for computing statistical quantities. In this paper, a Reduced-Order Model (ROM) based upon Proper Orthogonal Decomposition (POD) is introduced to circumvent this issue. First, an adjoint-based error estimation procedure is verified using a manufactured solution. Then a ROM-driven AMR strategy is studied using a LES model problem based on the 1D unsteady Burgers equation. Numerical results demonstrate that using ROMs not only lowers storage requirements, but also has no impact on the effectiveness of adjoint-based AMR.
\end{abstract}

\section{INTRODUCTION}

Large Eddy Simulation (LES), in which one resolves large-scale turbulent structures while modelling the impact from smaller turbulent scales, has the potential to deliver reliable flow predictions for many applications, such as those involving flow separation from a smooth surface or the analysis of acoustic sources [1]. LES has been shown to be highly successful in situations where the computational mesh is fine enough to resolve an appropriate range of large scales [2]. Nonetheless, defining such a computational mesh for obtaining an accurate solution with tractable computing cost is a challenging task, particularly in practical applications with complex geometries and unknown physical features.

In practice, the construction of such meshes usually involves trial and error, even for engineering experts. It is thus natural to consider Adaptive Mesh Refinement (AMR) techniques [3] in order to automatically construct the computational mesh. Physical features, such as wall distance, vorticity or shocks, have been widely used to guide AMR. However, local features are not necessarily related to the physical quantity of interest (QoI). Consequently, adjoint methods have been proposed to connect the local mesh contribution with the error in a chosen QoI, so that the adapted mesh can provide the highest accuracy per degree of freedom. This approach has been successfully used in both steady laminar and turbulent 
flow problems [4].

When adjoint-based AMR is employed for LES, there are complications. In unsteady simulations, the primal flow problem is advanced forward in time while the adjoint problem needs to be solved backward in time. Due to the non-linearity of Navier-Stokes equations, flow states from the primal LES are required for solving the adjoint problem. For small problems, we can store complete primal flow solutions in memory and then use this data to solve the adjoint problem. For practical problems, however, we are normally interested in QoIs computed over long averaging times, leading to prohibitive memory requirements. In order to handle this difficulty, some researchers choose to store the primal solution on hard disks $[5,6,7,8]$. However, the speed of data communication between hard disk and solver is much slower than the access to main memory. Others choose to store flow solution snapshots at a prescribed frequency and interpolate intermediate values in time $[9,10]$, which has also been employed in high-Re number problems [11]. Another solution is to apply the checkpointing technique [12] in which optimalselected primal states are stored and then used to re-solve the primal problem locally. Such techniques have been employed to solve unsteady adjoint problem in sensitivity analyses of dynamical systems [13], cylinder flows [14], and tonal noise [15]. However, this requires significant computational effort due to the re-computation. In order to make adjoint-based AMR affordable and efficient for LES, we consider a reduced-order model (ROM), based on proper orthogonal decomposition (POD), to circumvent these difficulties.

This paper is organized as follows. After a problem formulation in Section 2, on which the variational multiscale method and numerical discretization of LES are presented, the associated adjoint equation is

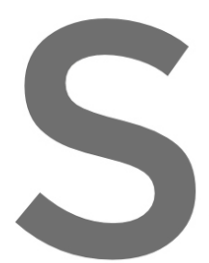
derived in Section 3 anc and procedure of mesh ac described in Section 5. solutions in Section 6, unsteady viscous Burg Section 7.
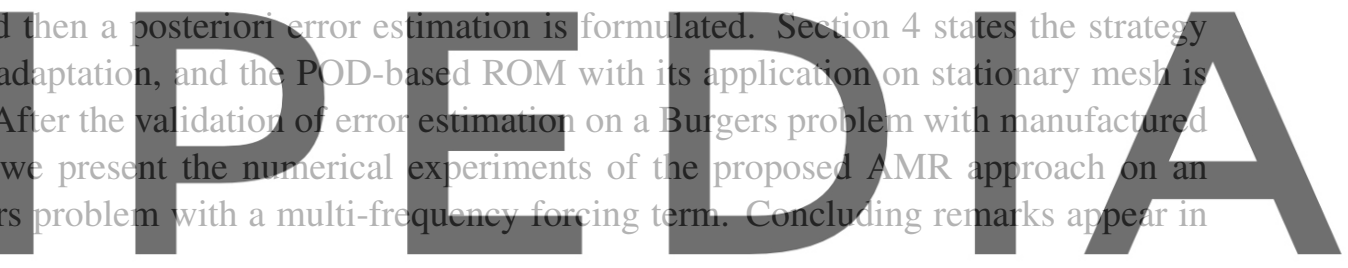

\section{Register for freelatihatpo/kwwmscipedia.com to download the version without the watermark}

We consider the one-dimensional (1D) Burgers equation over a space-time domain $\Omega:[0,1] \times \mathrm{I}:[0, \mathrm{~T}]$. The Burgers equation is often used as a mathematical model for the applications which involve shock wave propagation in viscous flows and turbulence [16]. The Burgers equation is expressed as

$$
\mathcal{N}(u)=\frac{\partial u}{\partial t}+u \frac{\partial u}{\partial x}-v \frac{\partial^{2} u}{\partial x^{2}}=f
$$

where $\mathcal{N}(\cdot)$ is a nonlinear operator, and $u$ is the solution with the boundary conditions $u(0, t)=u(1, t)=0$ and initial condition $u(x, 0)=u_{t_{0}} . v$ is the viscosity coefficient and $f \in \mathbb{R}$ is a given forcing term. Note that we use a $1 \mathrm{D}$ problem here to explore the methodology. However, approaches considered can be directly extended to multi-dimensional flow problems.

\subsection{Variational multiscale method}

We employ the Finite Element Method (FEM) to solve the flow problem in weak form

$$
\mathcal{R}(u, \omega)=\left(u_{t}, \omega\right)-\left(u u / 2, \omega_{x}\right)+\left(v u_{x}, \omega_{x}\right)-(f, \omega)=0,
$$


for $\forall \omega \in \mathcal{V}$, where $\mathcal{R}(\cdot, \cdot)$ is the residual operator in weak form. $\omega \in \mathcal{V}$ represents the weighting functions and $\mathcal{V}=\mathcal{V}(\Omega)$ denotes both the solution space and weighting space. $(\cdot, \cdot)$ is the $L_{2}$ inner product. The inner product used in this paper is defined on the spatial domain $\Omega$ by default.

The Variational Multiscale Method (VMM) is utilized as a sub-grid model for LES. In VMM, the flow solution is split into two components, the resolved scales $\bar{u}$ and the unresolved scales $u^{\prime}$. The unresolved scale equations are driven by the strong residual, i.e. $R(\bar{u})=\mathcal{N}(\bar{u})-f$. The simplest algebraic model for $u^{\prime}$ uses a volume-averaged Green's function to write $u^{\prime}=-\tau R(\bar{u})$. For this problem, we use an expression for $\tau$ from Z. Wang and Oberai [17], viz. $\tau=\left[\frac{4}{h^{2}} u^{2}+3 \pi v^{2}\left(\frac{4}{h^{2}}\right)^{2}\right]^{-1 / 2}$. Substituting $u=\bar{u}+u^{\prime}$ into Equation (2), the expression of weak form is stated as

$$
\mathcal{A}(\bar{u}, \omega)=\left(\bar{u}_{t}, \omega\right)-\left(\frac{1}{2} \bar{u} \bar{u}, \omega_{x}\right)+\left(v \bar{u}_{x}, \omega_{x}\right)-(f, \omega)-\left(\bar{u} u^{\prime}, \omega_{x}\right)-\left(\frac{1}{2} u^{\prime 2}, \omega_{x}\right)-\left(v u^{\prime}, \omega_{x x}\right)=0 .
$$

By virtue of $u^{\prime}=-\tau R(\bar{u})$, the $\bar{u}$ is exclusively determined from Equation (3).

\subsection{Discretization}

When numerically solving the flow problem, we replace $\bar{u}$ by $\bar{u}_{h}$, leading to the discrete system

$$
\mathcal{A}_{h}\left(\bar{u}_{h}, \omega_{h}\right)=0, \forall \omega_{h} \in \mathcal{V}_{h},
$$

where the subscript $h$ denotes the grid size of computational mesh with $\mathrm{N}$ degree of freedom. $\bar{u}_{h} \in \mathcal{V}_{h}$ is abbreviated to $u_{h}$ in the subsequent content if there is no conflict. The semi-discrete technique is utilized to discretize this unsteady model problem. Specifically, we use piecewise linear basis functions

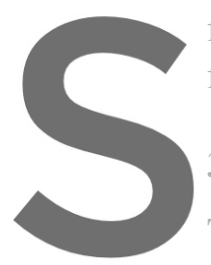

for spatial discretization

from $t=0$ to $t=T$.

3 ADJOINT-BASEID ERROR ESTIMATION

The adjoint method is used to provide estimates
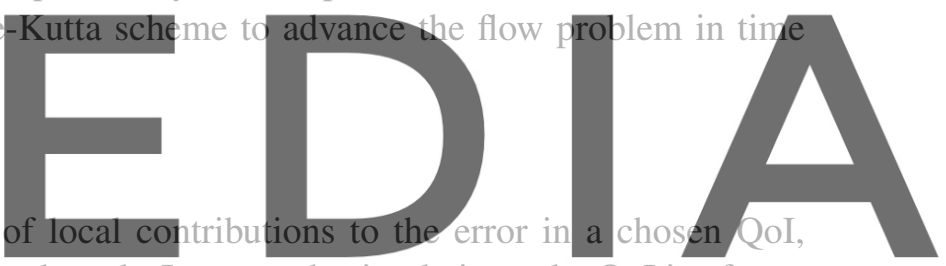

allowing for the construction of goal-oriented adapted mesh. In unsteady simulations, the QoI is often a

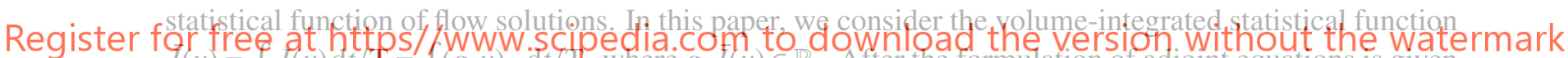
$g(u)=\int_{I}(u) \mathrm{d} t / T=\int_{I}(g, u)_{\Omega} \mathrm{d} t / 1$, where $g, f(u) \in \mathbb{R}$. After the formulation of adjoint equations is given in this section, a framework to a posterior error estimation is obtained, which will be used to guide the mesh refinement in Section 4.

\subsection{Adjoint formulation}

By virtue of a Lagrange function, the adjoint equation is derived for the Burgers problem as follows

$$
\mathcal{L}_{u}^{*} v=-\frac{\partial v}{\partial t}-u \frac{\partial v}{\partial x}-v \frac{\partial^{2} v}{\partial x^{2}}=g_{u},
$$

where $v$ is the Lagrange multiplier or adjoint variable with homogeneous boundary conditions $v_{x=0}=$ $v_{x=1}=0$ and initial condition $v_{t=\mathrm{T}}=0 . \mathcal{L}_{u}^{*}(\cdot)$ is a linear adjoint operator which relies on the primal flow solution $u . g_{u}$ is a Fréchét derivative of $J(u)$, defined as

$$
\left(g_{u}, \tilde{u}\right)_{\Omega}:=\lim _{\epsilon \rightarrow 0} \frac{J(u+\epsilon \tilde{u})-J(u)}{\epsilon}, \quad \forall u, \tilde{u} \in \mathcal{V} .
$$

The adjoint residual operator is expressed as $R_{[u]}^{*}(\cdot)=\mathcal{L}_{u}^{*}(\cdot)-g_{u}$. We solve the adjoint problem using the same VMM used for the primal flow problem. 


\subsection{A posteriori error estimation framework}

Before developing a posteriori error estimation, we consider the adjoint identity and averaging operator. The adjoint identity, which can be easily verified by partial integration, is

$$
\left(\mathcal{L}_{u}^{*} v, \tilde{u}\right)_{\Omega \times \mathrm{I}}=\left(v, \mathcal{L}_{u} \tilde{u}\right)_{\Omega \times \mathrm{I}}+\left.(v, \tilde{u})_{\Omega}\right|_{t=t_{0}},
$$

for $\forall v, u, \tilde{u} \in \mathcal{V}$. The last term is the contribution of non-zero values at starting time $t_{0}$. $\mathcal{L}_{u}$ is the Fréchét derivative of $\mathcal{N}(u)$

$$
\mathcal{L}_{u} \tilde{u}:=\lim _{\epsilon \rightarrow 0} \frac{\mathcal{N}(u+\epsilon \tilde{u})-\mathcal{N}((u)}{\epsilon}, \quad \forall u, \tilde{u} \in \mathcal{V} .
$$

We can formulate the averaging differential operator, $\overline{\mathcal{L}}_{\left(u_{1}, u_{2}\right)}^{*}(\cdot)$, by integrating the adjoint equation (5), enabling us to estimate the error for nonlinear problems. By defining $u=u_{1}+\theta\left(u_{2}-u_{1}\right)$, the adjoint governing equation is integrated from $u_{1}$ to $u_{2}$

$$
\overline{\mathcal{L}}_{\left(u_{1}, u_{2}\right)}^{*} v \equiv \int_{0}^{1} \mathcal{L}_{u=u_{1}+\theta\left(u_{2}-u_{1}\right)}^{*} v \mathrm{~d} \theta \stackrel{\text { Equation (5) }}{=} \int_{0}^{1} g_{u=u_{1}+\theta\left(u_{2}-u_{1}\right)} \mathrm{d} \theta \equiv \bar{g}_{\left(u_{1}, u_{2}\right)},
$$

where the subscript denotes the integration domain and $\bar{g}_{\left(u_{1}, u_{2}\right)}$ is averaged value of $g_{u}$. Then we can have the weak form as $\left(\overline{\mathcal{L}}_{\left(u, u_{h}\right)}^{*} v, \omega\right)=\left(\bar{g}_{\left(u, u_{h}\right)}, \omega\right), \forall \omega \in \mathcal{V}$.

Likewise, the average linearized operator in Equation (9) maintains the same property of adjoint identity as follows

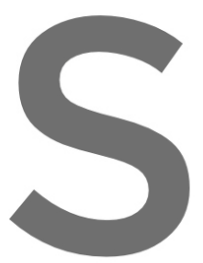

where $v, \bar{u}, u_{1}, u_{2} \in \mathcal{V}$

Based on the definition
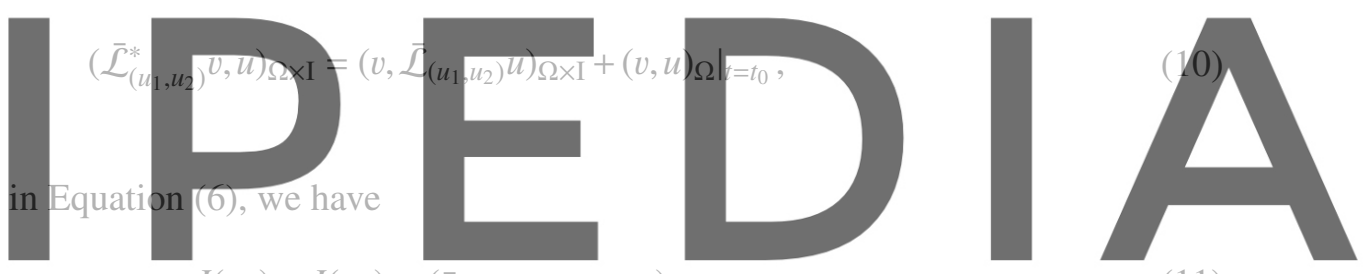

$$
J\left(u_{1}\right)-J\left(u_{2}\right)=\left(\bar{g}_{\left(u_{1}, u_{2}\right)}, u_{1}-u_{2}\right) .
$$

(11)

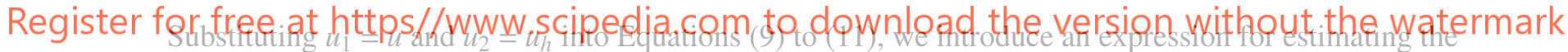
error $\epsilon$ of the QoI

$$
\epsilon=\bar{J}(u)-\bar{J}\left(u_{h}\right)=\underbrace{-\frac{1}{\mathrm{~T}}\left(v_{h}, R\left(u_{h}\right)\right)_{\Omega \times \mathrm{I}}}_{\text {Adjoint correction }}+\underbrace{\frac{1}{\mathrm{~T}}\left(R_{\left[\frac{u+u_{h}}{2}\right]}^{*}\left(v_{h}\right), u_{h}-u\right)_{\Omega \times \mathrm{I}}}_{\text {Remaining error }}-\left.\frac{1}{\mathrm{~T}}\left(v_{h}, u_{h}-u\right)_{\Omega}\right|_{t=t_{0}}=\sum_{e}^{\mathrm{N}} \epsilon_{e} .
$$

The error estimation is expressed as an adjoint correction, remaining error and temporal contribution. The temporal contribution is due to the non-zero value of the initial condition during the statistical time period, which disappears in steady problems. In practice, $R_{\left[\frac{u+u_{h}}{2}\right]}^{*}\left(v_{h}\right)$ is replaced by $R_{\left[u_{h}\right]}^{*}\left(v_{h}\right)$, where $u_{h}$ is the discrete flow solution. Here, the VMM is used to evaluate the exact solution $u$ as $u_{h}+u_{h}^{\prime}$. Note that the error estimation can be constrained to each element as shown in the last part of the equation, which is expressed in terms of elemental error estimators $\epsilon_{e}$. 


\section{MESH ADAPTATION}

To facilitate mesh adaptation, an error indicator $\eta_{e}$ is determined from the elemental error estimator as $\eta_{e}=\left|\epsilon_{e}\right|$. Different refinement strategies [4] can be formulated based on $\eta_{e}$. We employ a prescribed percentage (10\%) of mesh elements with largest errors as the criterion for mesh refinement. The resulting procedure of adjoint-based AMR is presented in Figure 1. In each AMR loop, the error estimation and adaptation strategy are executed to generate a new computational mesh for the next AMR level.

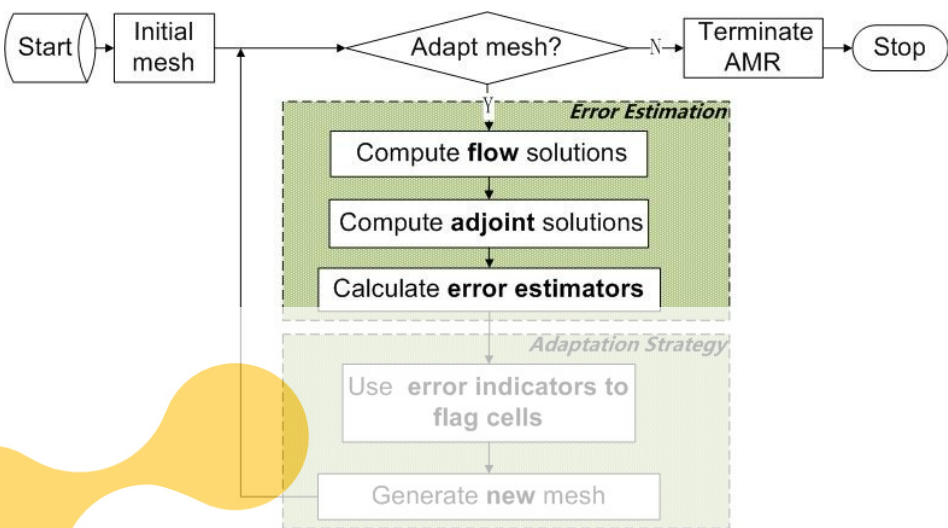

Figure 1: A general procedure of mesh adaptation based on adjoint method for unsteady simulations

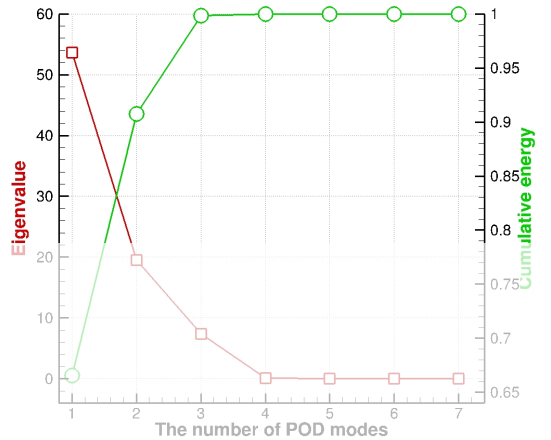

Figure 2: POD analyses of a Burgers problem solved with a stationary mesh

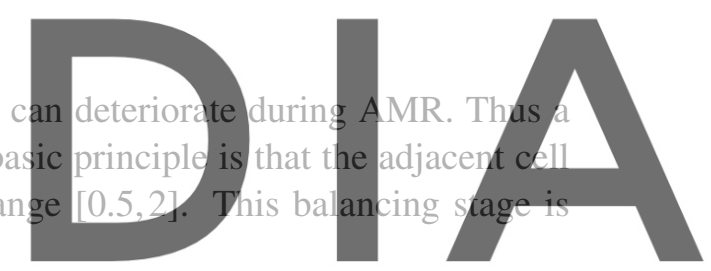

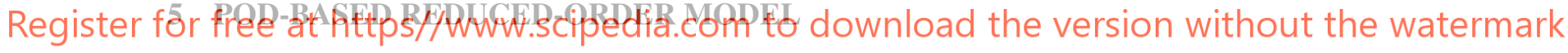

In order to make the adjoint-based AMR affordable for LES, a reduced-order model is introduced to represent the flow solution in a low-order space, which can be solved and accessed efficiently. Generally, there are different types of ROMs in literature, including interpolated ROM and projected ROM. We consider the former type because it is unnecessary to predict the future solution in this case. The ROM is constructed using proper orthogonal decomposition (POD), which is achieved by singular value decomposition (SVD) implemented using the LAPACK library package [18]. Figure 2 presents the eigenvalues and cumulative energy of the solutions to a Burgers problem. In this case, the first mode represents a significant part of the instantaneous solution as it accounts for $66.5 \%$ of the total energy.

\section{NUMERICAL EXPERIMENTS}

\subsection{Verification in a manufactured solution}

First of all, the error estimation procedure is verified using a manufactured solution, $u(x, t)=\sin ^{2}(\pi t)$ $\sin (\pi x)$. This exact solution is used to compute the actual value of the QoI in different AMR levels, so that the error estimates based on VMM and adjoint solutions can be evaluated. The discrete solutions of 
primal and adjoint problems are shown for $v=0.01$ in Figure 3(a). Figure 3(b) depicts the approximation of the QoI and corresponding error. The computed QoI converges to the exact one as the mesh is uniformly refined, and the error estimation has a good agreement with the actual value.
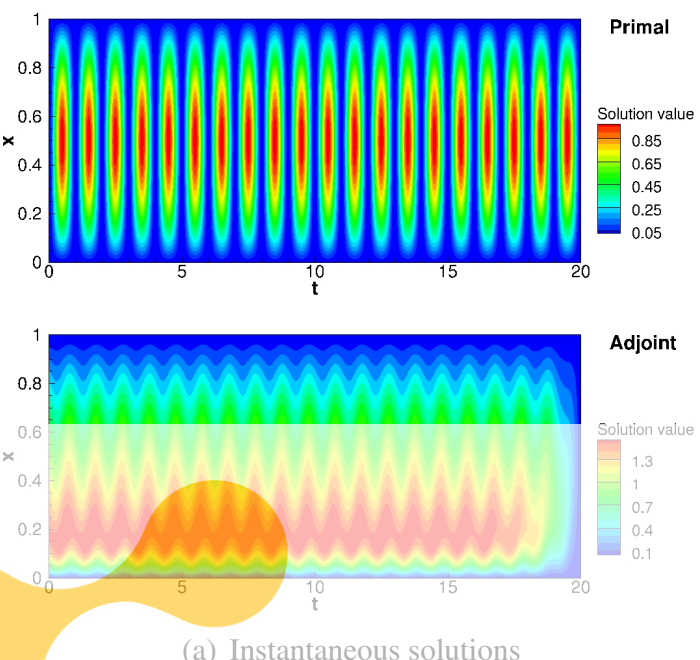

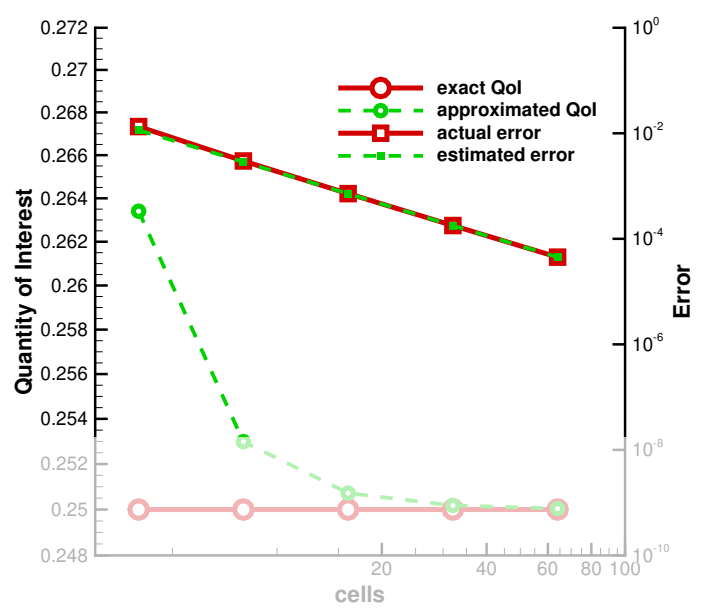

(b) Error estimation

Figure 3: Instantaneous solutions for a manufactured Burgers problem and associated error analysis of a volumeintegrated output on uniform refined meshes. Solid lines denote the values computed from analytical solutions while dash lines are the approximation from numerical solutions and the adjoint-based error estimation with VMM.
We now introduce a forcing term f for $v=0.01$ to produce the solution with farge fluctuations and strong flow gradients near the right boundary

Register for free at https//www.scipedia.com to download the version without the watermark

$$
f(x, t)=1+q(x) * \sum_{i=1}^{N} g_{i}(t) \sin \left(k_{i} x\right),
$$

where $N=3$ and the $g_{i}(t)$ are chosen so $\left|g_{i}(t)\right| \leq 1$

$$
g_{1}(t)=\sin (\pi t), k_{1}=\pi, g_{2}(t)=\sin (2 \pi t), k_{2}=2 \pi, g_{2}(t)=\sin (3 \pi t), k_{3}=3 \pi .
$$

$q(x)=5 / 30$ is a coefficient used to tune the amplitude of the forcing term at various wave numbers so that the flow fluctuations can be controlled independently. The primal problem is advanced froward from $t=0$ to $t=20$ while the adjoint problems is solved backward from $t=20$ to $t=10$. This temporal interval $([0,10])$ is sufficient long to allow the primal flow problem to arrive to a statistically steady state. Instantaneous solutions are shown in Figure 4.

The mesh adaptation strategy in Section 4 is compared with traditional uniform mesh refinement for this case. The reference value of the QoI is calculated on a fine mesh with 256 elements as there is no analytical solution. Figure 5(a) presents the computation of QoI from different methods and their associated errors. The QoI's computation from proposed AMR technique is clearly more accurate than 

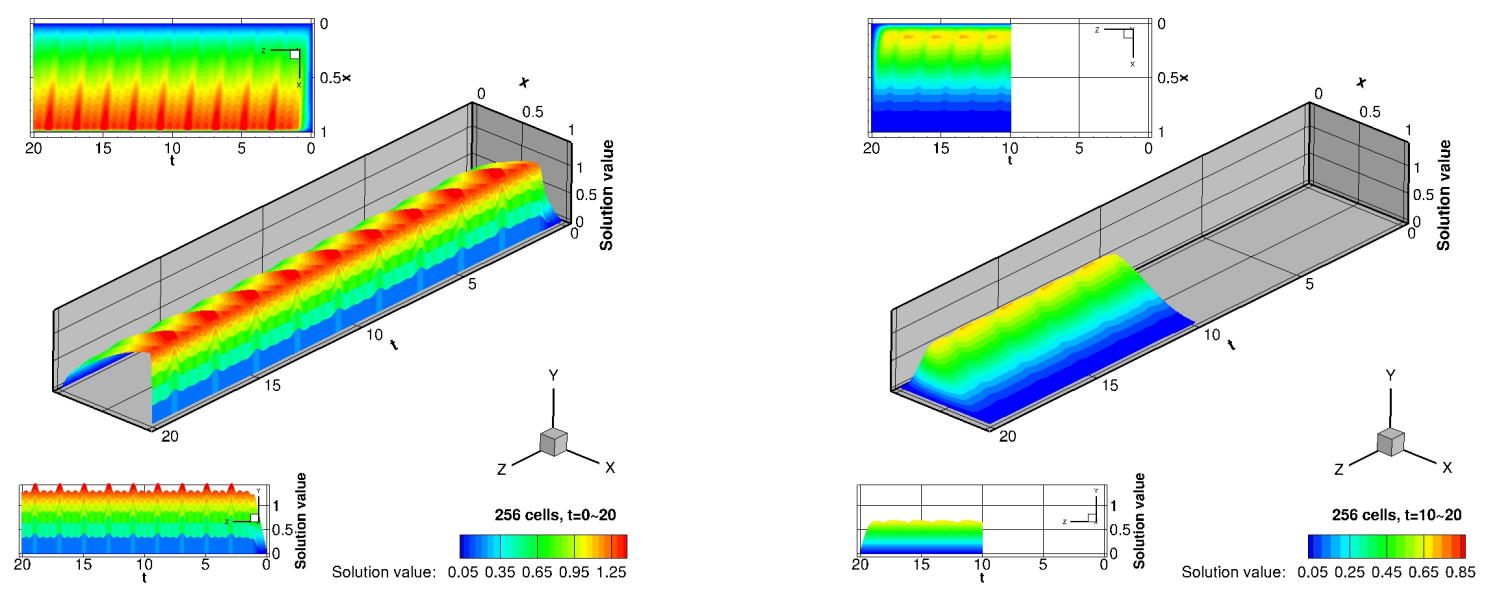

Figure 4: The distribution of (a) primal and (b) adjoint solutions from Burgers problems with a forcing term

uniform enrichment for a given number of degrees of freedom and the corresponding error also converges faster.

\subsection{ROM-driven mesh refinement}
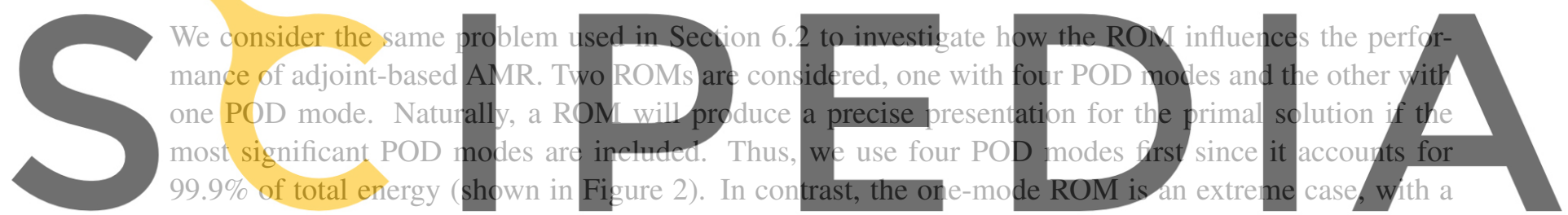

less accurate reconstruction of the primal solution but still able to reproduce its dominant features. The

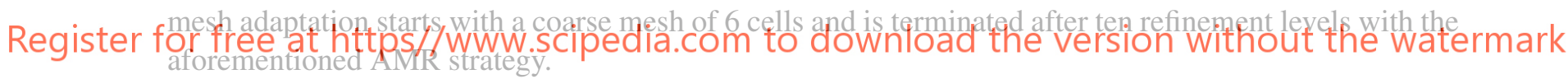

\subsubsection{ROM with four POD modes}

The computation of the QoI from ROM-driven AMR compared to full-order AMR is shown in Figure 5(a). They converge to the actual value in a quantitatively similar way, as does their approximating error as shown in Figure 5(b). Figure 6 demonstrates that the mesh refinement patterns computed based on the four-mode ROM agree well with those from full-order primal flow solutions.

Figure 7(a) depicts the development of cumulative energy within various AMR levels. Most notably from the standpoint of POD analysis, four POD modes can still capture more than $99.9 \%$ of the total energy even for the fine meshes, which are obtained during later process of AMR. Thus four-mode ROM-driven AMR does not behave differently from the standard AMR employing the full-order $u_{h}$. 

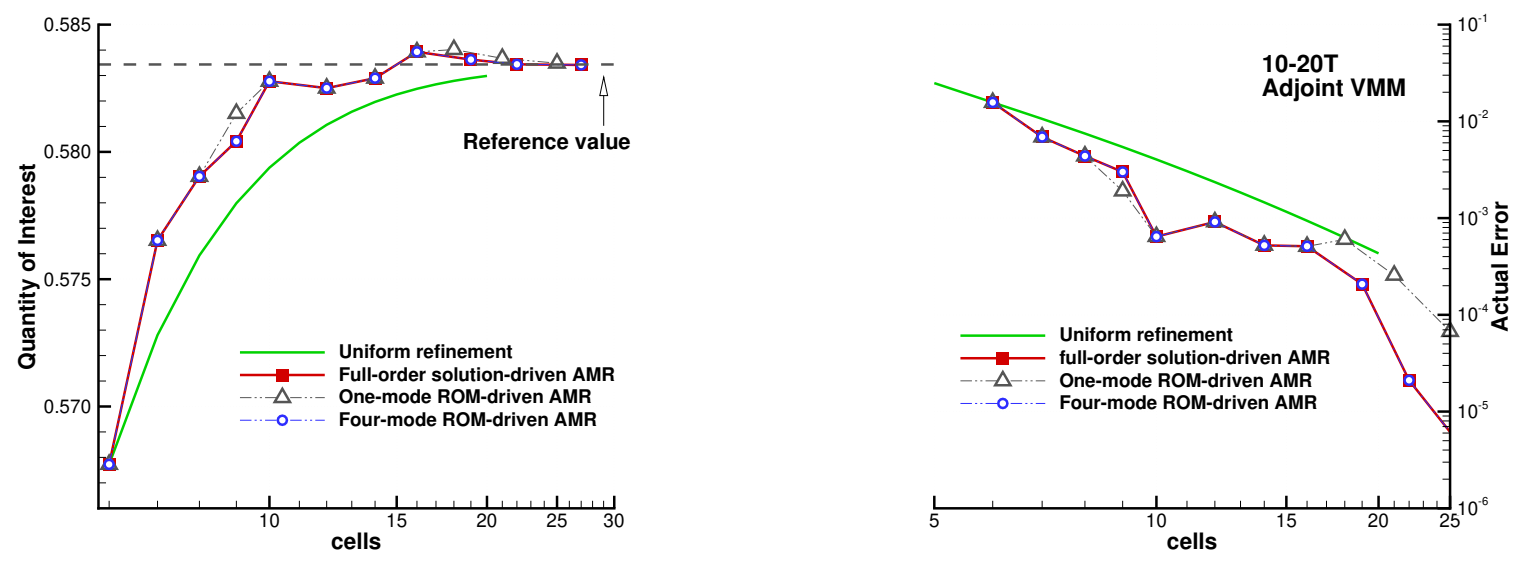

Figure 5: Comparisons of (a) the approximation of QoI and (b) associated approximating error between uniform refinement $(-)$, and AMR based on full-order flow solutions (- - ), four-mode ROM (- $\mathrm{O}^{--}$) and one-mode ROM (-..-..-), respectively.
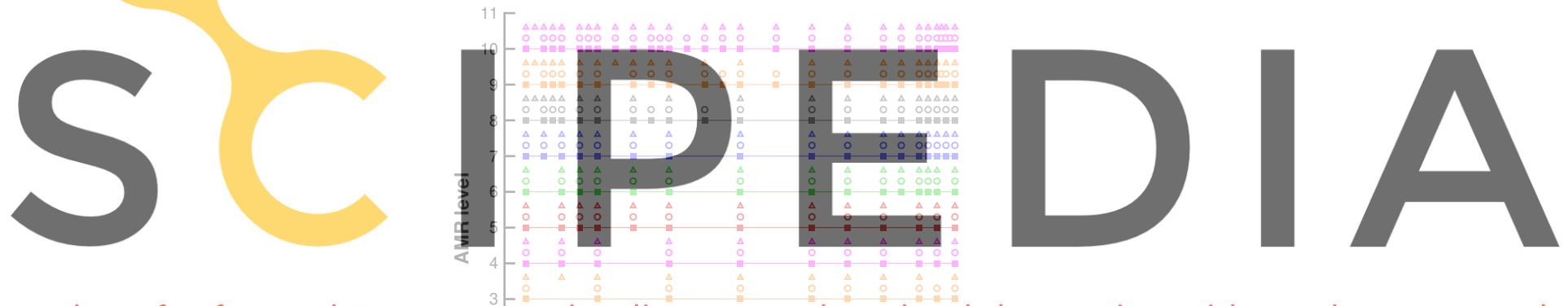

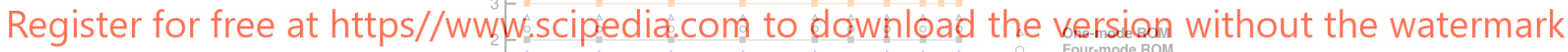

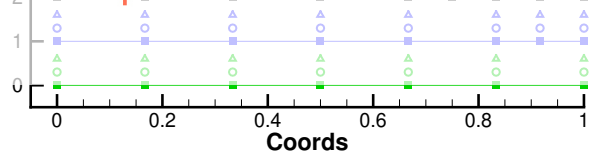

Figure 6: Comparisons of mesh refinement patterns from AMR based on flow solution ( - ), four-mode ROM (o) and one-mode ROM $(\Delta)$, respectively. Each color denotes one AMR level.

\subsubsection{ROM with one POD mode}

We now study an extreme situation with only one mode to construct a ROM. As before, Figure 5 shows the convergence of QoI and their corresponding errors using a one-mode ROM. The QoI's convergence from a one-mode ROM is quite similar to those from full-order primal solutions although there are differences at some AMR levels. But it is still much better than uniform refinement. From the mesh refinement patterns shown in Figure 6, we can observe that the mesh refined from a one-mode ROM is not completely the same as those based on full-order primal solutions. When comparing different 


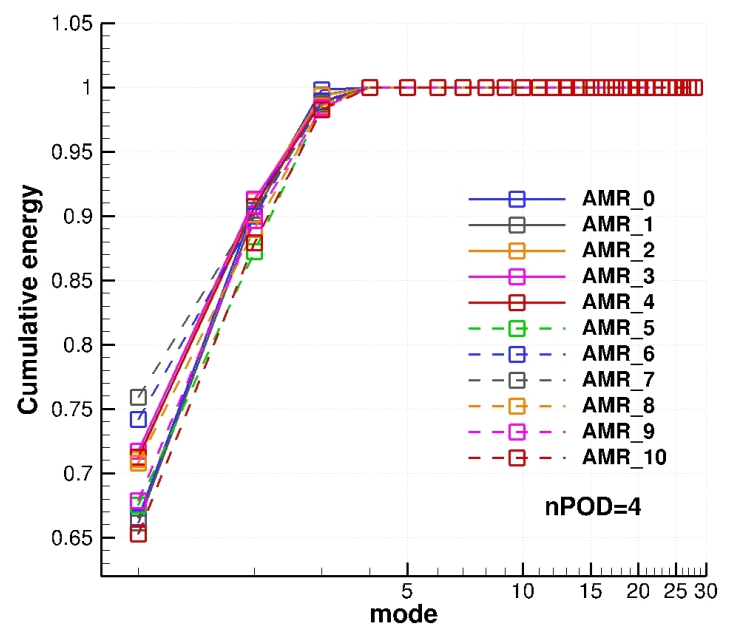

(a) Four-mode ROM

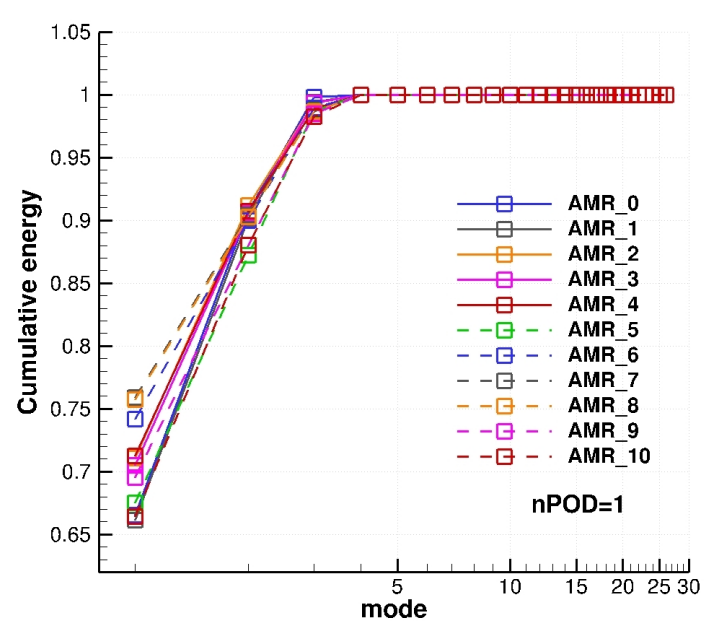

(b) One-mode ROM

Figure 7: Comparisons of cumulative energy during different AMR levels computed with (a) four-mode ROM and (b) one-mode ROM, respectively.

adapted meshes, we can note that the low-order ROM changes the AMR order in coarse mesh regions (e.g. 3rd AMR level) but can reach the same computing mesh later (e.g. 4th AMR level). On the other hand, the approximating error in tater AMR stages is affected by using onty one mode when the mesh becomes fine in space (see in Figure 7(b) and the fi

Figure 8 presents discrete adjoint solutions on two der AMR, a coarse mesh in Figure 8 (a) and a fine mesh ilr. based on the full-order primal solution, a four-mode ROM and a one-mode ROM, respectively. On the coarse mesh, the one-mode ROM is able to produce adjoint solutions with both features and magnitudes

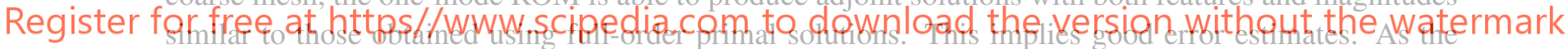

AMR proceeds to finer meshes, the one-mode ROM is unable to present high-frequency information and thus produces relative smooth adjoint solutions. The high-frequency components of primal flow solutions, however, become increasingly important for obtaining precise error estimates on fine meshes. This leads to deterioration in the error estimate from the one-mode ROM. Conversely, the four-mode ROM includes both low and high frequency information and provides good estimates on both computational meshes.

Overall, the ROM-driven AMR can preserve the efficiency of solution-driven adjoint-based AMR in the current case even with only a single POD mode. This may be due to a relative insensitivity of the chosen QoI to high-frequency solution components, which tend to be smaller magnitudes in the current problem. However, this is not necessarily constrictive for LES applications, where large scales contain the majority of the flows' energy, and we are often interested in the relatively smooth QoIs such as mean profiles. 

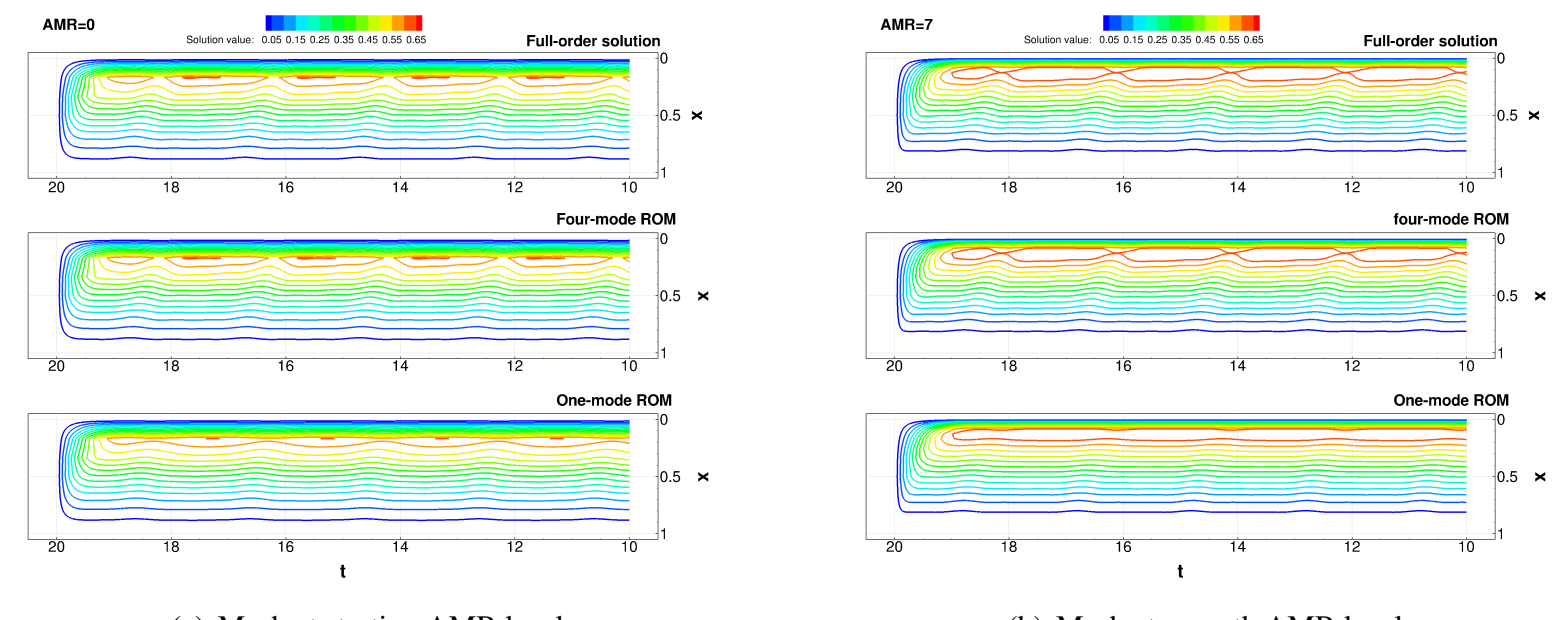

(a) Mesh at starting AMR level

(b) Mesh at seventh AMR level

Figure 8: Comparisons of discrete adjoint solutions at two different AMR meshes, computed based on full-order flow solution, four-mode ROM and one-mode ROM, respectively.

\section{CONCLUSIONS}

We have developed a ROM-driven adjoint-based mesh adaptation strategy for unsteady problems. The effectiveness of the error estimation based on the adjoint solution and the VMM has been verified using an unsteady Burgers problem with a manufactured encountered when solving introduced to represent using a Burgers problem full-order primal solution is shown to be more ef
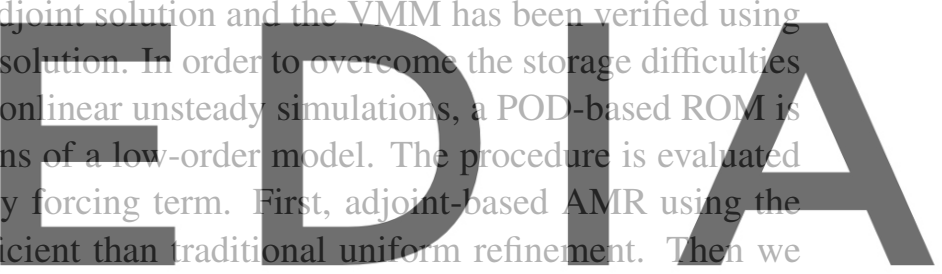

demonstrate that using the ROM for the primal solution does not significantly affect the performance of

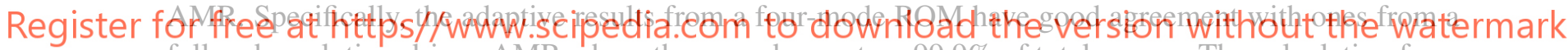
full-order solution-driven AMR where these modes capture $99.9 \%$ of total energy. The calculation from a one-mode ROM still considerably outperforms uniform refinement as the first POD mode accounts for $65-75 \%$ of total energy during AMR. Furthermore, a one-mode ROM is able to capture the essential part of the solution on coarse meshes. As expected, using a ROM with sufficient modes does not affect the adjoint-based AMR. Using a very low-order ROM, however, can still be effective as least when the calculation of the QoI is not dependent on the smaller scales of primal solutions. These results indicate that the proposed AMR strategy will likely be useful for more challenging problems.

\section{ACKNOWLEDGEMENTS}

The authors would like to thank the financial support by China Scholarship Council.

\section{REFERENCES}

[1] Georgiadis, N.J., Rizzetta, D.P., and Fureby, C. Large-Eddy Simulation: Current Capabilities, Recommended Practices, and Future Research. AIAA Journal. (2010) 48 (8):1772-1784. 
[2] Larsson, J. and Wang, Q. The prospect of using large eddy and detached eddy simulations in engineering design, and the research required to get there. Philosophical Transactions of the Royal Society A: Mathematical, Physical and Engineering Sciences. (2014) 372 (2022):20130329-20130329.

[3] Slotnick, J. et al. CFD vision 2030 study: a path to revolutionary computational aerosciences. Technical Report NASA/CR-2014-218178. Washington, DC 20546-0001: National Aeronautics and Space Administration, (2014), p. 58.

[4] Fidkowski, K.J. and Darmofal, D.L. Review of Output-Based Error Estimation and Mesh Adaptation in Computational Fluid Dynamics. AIAA Journal. (2011) 49 (4):673-694.

[5] Besier, M. and Rannacher, R. Goal-oriented space-time adaptivity in the finite element Galerkin method for the computation of nonstationary incompressible flow. International Journal for $\mathrm{Nu}$ merical Methods in Fluids. (2012) 70 (9):1139-1166.

[6] Fidkowski, K.J. and Luo, Y. Output-based space-time mesh adaptation for the compressible NavierStokes equations. Journal of Computational Physics. (2011) 230 (14):5753-5773.

[7] Schmich, M. and Vexler, B. Adaptivity with Dynamic Meshes for Space-Time Finite Element Discretizations of Parabolic Equations. SIAM Journal on Scientific Computing. (2008) 30 (1):369393.

[8] Flynt, B. and Mavriplis, D. Discrete Adjoint Based Adaptive Error Control in Unsteady Flow Problems. 50th AIAA Aerospace Sciences Meeting including the New Horizons Forum and Aerospace Exposition. 50th AIAA Aerospace Sciences Meeting including the New Horizons Forum and Aerospace Exposition. Nashville, Tennessee: American Institute of Aeronautics and Astronautics, (2012).

[9] Hoffman, J. et al. Towards a parameter-free method for high Reynolds number turbulent flow simulation based on adaptive finite element approximation. Computer Methods in Applied Mechanics and Engineering. (2015) 288:60-74.

[10] Hoffman, J. and Johnson, C. A new approach to computational turbulence modeling. Computer Methods in Applied Mechanics and Engineering. (2006) 195 (23):2865-2880.

[11] Jansson, J. et al. Time-Resolved Adaptive Direct FEM Simulation of High-Lift Aircraft Configurations. Numerical Simulation of the Aerodynamics of High-Lift Configurations. Ed. by O.D. López Mejia and J.A. Escobar Gomez. Cham: Springer International Publishing, (2018), p. 6792.

[12] Wang, Q., Moin, P., and Iaccarino, G. Minimal Repetition Dynamic Checkpointing Algorithm for Unsteady Adjoint Calculation. SIAM Journal on Scientific Computing. (2009) 31 (4):2549-2567.

[13] Wang, Q. Forward and adjoint sensitivity computation of chaotic dynamical systems. Journal of Computational Physics. (2013) 235:1-13.

[14] Wang, Q. and Gao, J.-H. The drag-adjoint field of a circular cylinder wake at Reynolds numbers 20, 100 and 500. Journal of Fluid Mechanics. (2013) 730:145-161.

[15] Fosas de Pando, M., Schmid, P.J., and Sipp, D. On the receptivity of aerofoil tonal noise: an adjoint analysis. Journal of Fluid Mechanics. (2017) 812:771-791.

[16] Caldwell, J., Wanless, P., and Cook, A.E. A finite element approach to Burgers' equation. Applied Mathematical Modelling. (1981) 5 (3):189-193.

[17] Wang, Z. and Oberai, A. Spectral analysis of the dissipation of the residual-based variational multiscale method. Computer Methods in Applied Mechanics and Engineering. (2010) 199 (13):810818. 
[18] Taira, K. et al. Modal Analysis of Fluid Flows: An Overview. AIAA Journal. (2017) 55 (12):40134041. 